\title{
Investigating the Validity of Acoustic Spectrum as a Prediction Tool for Pump Cavitation
}

\author{
Ashraf Kotb, Abdulaziz Morgan Abdulaziz \\ Department of Mechanical Power Engineering, Faculty of Engineering, Ain Shams University, Cairo, Egypt \\ Email: Ashraf Ibrahim@eng.asu.edu.eg, Abdelaziz_Morgan@eng.asu.edu.eg
}

Received 18 September 2015; accepted 12 December 2015; published 15 December 2015

Copyright $@ 2015$ by authors and Scientific Research Publishing Inc.

This work is licensed under the Creative Commons Attribution International License (CC BY). http://creativecommons.org/licenses/by/4.0/

(c) (i) Open Access

\begin{abstract}
Cavitation in pumps causes destructive consequences; it must be detected and prevented. The aim of the present work is investigating the validity of sound spectrum as a prediction tool for pump cavitation. Results showed that; for the discrete frequencies of $R F=47.5 \mathrm{~Hz}$, and $\mathrm{BPF}=285 \mathrm{~Hz}$ and its second, third, and fourth harmonics of $570 \mathrm{~Hz}, 855 \mathrm{~Hz}$, and $1140 \mathrm{~Hz}$ respectively; there are no great variations in the noise signal for the cavitation and non-cavitation conditions. For the discrete frequency of $147 \mathrm{~Hz}$, there is also no great variation in the noise signal at this frequency. The only apparent result is that; the occurrence of cavitation results high energy noise signals at high frequencies from $1000 \mathrm{~Hz}$ to $10000 \mathrm{~Hz}$. The absence of any discrete frequency to be monitored makes the sound spectrum not valid as a prediction tool for cavitation in the pumps.
\end{abstract}

\section{Keywords}

Centrifugal Pump, Cavitation, Acoustic, Blade Passing Frequency

\section{Introduction}

It is expected that all pumps are subjected to cavitation. It occurs when the static pressure at the suction port or through the impeller falls below the saturation pressure corresponds to the fluid temperature. Pressure is reduced due to many aspects; one of the inherent aspects is the inlet guide vanes. At prewhirl angle of $24^{\circ}$, inlet guide vanes cause pressure drop of $7.6 \%$ at the suction pipe inlet [1].

Wherever the pressure falls, the fluid starts to flash and evaporate with the onset of bubbles where their number and size depend mainly on the value of pressure. The bubbles travel with the fluid flow and collapse whenever reach a point of high pressure at the blade surface. This collapse causes pressure fluctuation with maximum 
amplitude at the blade leading edge for cavitation flow is 2.54 times larger than that for non-cavitation flow, under large discharge, the magnification on the maximum pressure amplitude is 1.6 [2].

Cavitation causes destructive consequences as results of pressure fluctuation, audible sound, and vibration. It is associated with a non-recoverable degradation of the hydraulic performance, impeller pitting, and material erosion. Therefore cavitation in pumps must be detected and prevented.

There are several methods for detecting the incipient of cavitation; determination of the net positive suction head at a constant speed and flowrate, visualization of the inlet flow at the impeller eye, paint erosion on impeller blades, static pressure measurement in the flow, vibration and acoustic measurements.

Cavitation detection by acoustic measurements is based on understanding the sources of the generated noise from the pump.

Mechanical sources of noise include vibration of pump components as a result of; pressure variations through the pump flow passages, defective components such as impeller, seal rubs, bearings, unbalanced impellers, improper installation of pump driver and misalignment.

Hydraulic sources of noise include both the transient and instationarity of flow causes; Transients; during the startup and shutdown of the pump, opening or closing of servicing valves may cause severe pressure waves (water hammer) with high intensity of noise levels. Instationarity of flow; as a result of asymmetric outgoing flow of the impeller due to secondary flows in the rotating impeller, number of vanes and thickness which cause a notch in the wake flow, guide vanes and tongue in the pump casing. The instationarity of flow is a combination of both the effect of pressure pulsations and turbulence. Pressure pulsations depend on the design of impeller, diffuser, operating parameters, and the distance between impeller and the volute tongue. Turbulence induces vortices and wakes in the clearance between the impeller vane tips and the diffuser or volute lips. Pressure pulsations produced in this way impinge on the impeller and result in shaft vibrations and noise over a wide frequency range.

Also, the noise generated from the pump cavitation depends on the fluid temperature in the range from $80^{\circ} \mathrm{C}$ and $280^{\circ} \mathrm{C}$ for water [3].

In the case of pump cavitation, random, high frequency broadband energy, which is sometimes superimposed with the blade passing frequency harmonics are generated, the cavitation noise having a characteristic crackling or hissing noise.

Acoustic measurements in the audible range are rarely used as an engineering method, as the total noise level depends on the design and type of the pump, installation, mechanical auxiliaries, operating conditions, and strength of cavitation.

Using the noise to detect the cavitation in pumps is well known but limited on the high frequency range of ultrasound (above $20 \mathrm{kHz}$ ), while the noise is measured inside the pump. In the audible frequency range (from 20 $\mathrm{Hz}$ to $20 \mathrm{kHz}$ ), studies of cavitation noise are still in development stage with much care of the filtering out with the rotational frequency and harmonics.

Many researchers measured and analyzed the noise spectrum and paid attention to the background noise as a trial to find a specific discrete frequency(s) at which the noise level is varied as a result of cavitation.

For $5 \mathrm{~kW}$ pumping set with six semi-open impellers, Cudina found at $147 \mathrm{~Hz}$, there is 12 and $20 \mathrm{~dB}$ difference in the noise level between the incipient and fully developed cavitation, with recommendations of using the $147 \mathrm{~Hz}$ sound signal as a cavitation detector [4] [5].

For three pumping sets, centrifugal pump with six semi-open impellers, chemical centrifugal pump with six closed impellers, and water mixed-flow pump with five semi-open rotor blades. Cudina and Prezelj measured the noise at discrete frequencies in the audible range to detect the incipient and fully developed cavitation in pump. They found great enough difference in the noise level between the two conditions for various distance between the microphone and the pump, where they found a great difference in noise peak at the discrete frequency of $147 \mathrm{~Hz}$ [6] [7].

For two centrifugal pumps, the metal alloy one has six blades closed impeller and the plastic one has six blades semi-open impeller. Cernetic et al. found the discrete frequency for the plastic one at $148 \mathrm{~Hz}$ [8].

For closed impeller and semi-open impeller, Cernetic tested two the different centrifugal pumps and found that; the convenient range of frequency for cavitation detection is above $1000 \mathrm{~Hz}$ [9].

The research in this field extends to consider different measuring techniques and instrumentation, V. Bull et al. used a fiber-optic hydrophone to detect the acoustic emissions over frequency range of 15 - $20 \mathrm{MHz}$ [10]. Antonio Baldassarre et al. used image processing for visual analysis of cavitation conditions [11]. 
It is noted from the literature survey that; most of researchers focused on the values of the discrete frequencies at which the noise signal has great variation between the cavitation and non-cavitation conditions. This will lead to a recommendation of monitoring the noise generated from the pump at these discrete frequencies as a prediction tool for the cavitation inception. The main thrust of the present work is measuring and analyzing the sound spectrum for centrifugal pump under cavitation and non-cavitation conditions for five cases while the pump discharge and pump rotational speed are kept constant. It is an attempt to declare the validity of sound spectrum as a prediction tool for pump cavitation.

\section{Theoretical Background}

The static pressure along a stream line is governed by Bernoulli equation, Equation (1), where it decreases as a result of increasing the velocity.

Figure 1 shows a schematic distribution of the pressure along the pump impeller where it has a minimum value somewhere at the impeller eye. Cavitation Occurs when the pressure falls below the vapor pressure.

$$
\frac{p_{s}}{\rho g}+\frac{c^{2}}{2 g}+z+H=\text { constant }
$$

Net Positive Suction Head $\left(\mathrm{NPSH}_{\mathrm{A}}\right)$ is defined as the available total suction head at the pump inlet above the head corresponding to the vapor pressure at that temperature.

$$
\mathrm{NPSH}_{\mathrm{A}}=\frac{p_{s}-p_{v}}{\rho g}+\frac{c_{s}^{2}}{2 g}
$$

$\mathrm{NPSH}_{\mathrm{R}}$ equals $\mathrm{NPSH}_{\mathrm{A}}$ when the pump head deteriorates by $3 \%$ from the main characteristic curve.

Thoma cavitation number is defined by

$$
\sigma=\frac{\mathrm{NPSH}_{\mathrm{A}}}{\mathrm{H}}
$$

When the investigation of cavitation extends to different pump discharges, the total head will not be constant. During this investigation it is therefore considered more appropriate to propose a new parameter called the cavitation strength parameter:

$$
\xi=\frac{\mathrm{NPSH}_{\mathrm{R}}-\mathrm{NPSH}_{\mathrm{A}}}{\mathrm{NPSH}_{\mathrm{R}}} \%
$$

During cavitation, bubbles are formed in a low pressure media. As the fluid moves through the impeller blades, the pressure is increased by the amount of energy added. At a certain pressure level the bubbles collapse near the wall producing large range of sound spectrum which can be sensed by acoustic sensor in the time domain. Integration of the acoustic amplitude with time would give the overall sound level. To correlate sound levels with cavitation only, other factors must be eliminated.

To get a minimum vibration causes with the associated sound level, the flow must be tangent to the impeller blades at pump inlet. The design blade angle at the design flow rate is $\beta_{1}$ which corresponds to the flow velocity at the blade inlet, $c_{1}$. Referring to Figure 2(a) and Figure 2(b), as the inlet flow velocity increases, $c_{1}{ }_{1}^{\prime}$, there will be a shift angle $\Delta \beta_{1}$ for the flow relative velocity, $\mathrm{w}_{1}{ }^{1}$, at blade entrance. This will increase the vibration causes.
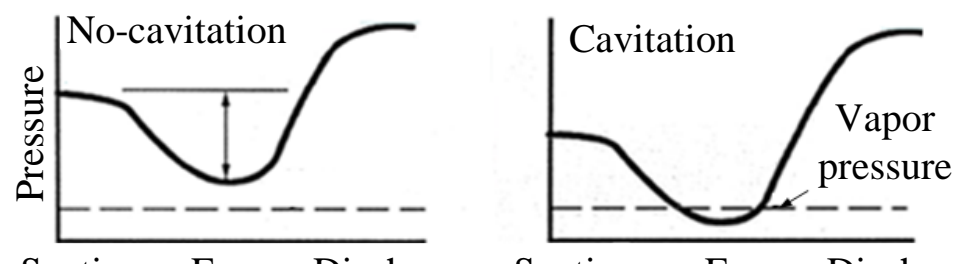

Suction Eye Discharge Suction Eye Discharge

Figure 1. Schematic distribution of the static pressure along the pump impeller. 


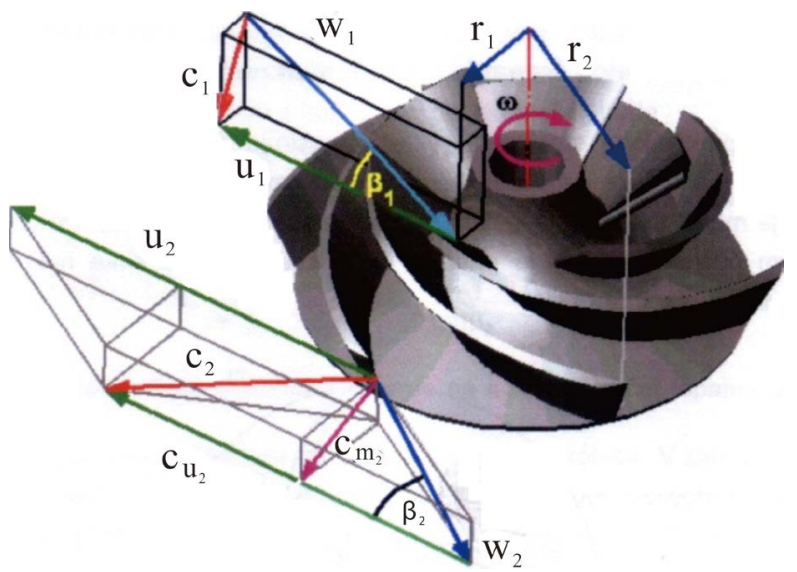

(a)

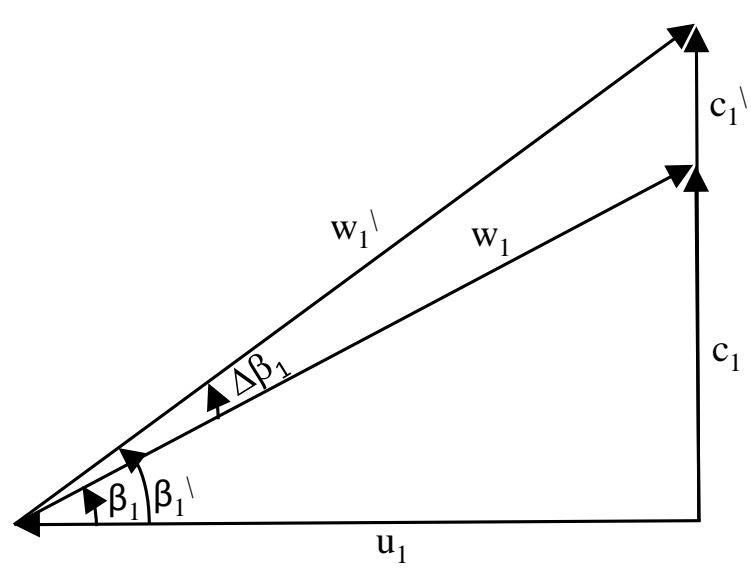

(b)

Figure 2. (a) Velocity triangles at the impeller inlet and outlet, (b) impeller blade inlet velocity triangle at two discharges. http://lib.convdocs.org/docs/index-126442.html?page=5.

Therefore, there will be a combined effect of both cavitation and flow inlet velocity on the sound level.

Analyzing the time-base acoustic signal with fast Fourier transform separates the discreet frequencies indicative to cavitation and other rotational effects. The impeller of a typical centrifugal pump has number of blades that rotates inside a volute casing. Each time any blade passes an arbitrary point on the pump, it generates small disturbance in the flow and consequently produces a pulse of vibration the discreet frequency called blade passing frequency, BPF. This frequency is equal to the impeller rotational speed multiplied by the number of blades.

\section{Materials and Methods}

The test rig in accordance with ISO 3555 and BS EN ISO 9906/2000 [12] specifications for acceptance tests for centrifugal, mixed flow and axial pumps Class B tests is shown in Figure 3(a), Figure 3(b). It is a closed loop water circuit composed of a pump, a tank, connecting PVC pipes and regulating valves. The pump rotational speed is precisely controlled with an electronic inverter within $0.1 \mathrm{~Hz}$ resolution. The test rig is provided with a set of measuring instrumentation for different variables. Water flow rate is measured by a pre-calibrated electromagnetic flow meter. The upstream and downstream pressures are measured by pre-calibrated dial gauges with additional pressure transducer; Keller Pa 21 SR, to record the downstream pressure. The sound measurements are made by GRAS free-field microphone type $46 \mathrm{AE}$, place $50 \mathrm{~cm}$ from the pump in the plane parallel to the pump axis as shown in Figure 3(c). The microphone band width is $3.5: 20,000 \mathrm{~Hz}$ with uncertainty of \pm 0.06 $\mathrm{dB}$ at the test frequency $250 \mathrm{~Hz}$. All measurements are acquired to a PC via the very specialized NI 9234 data acquisition card. Lab View software is used to simultaneously record the mass flow rate, downstream pressure, and sound levels. The software performs Fast Fourier Transform calculations.

The maximum uncertainty in measuring the mass flow rate as repeated 14 times for each test case is $0.5 \%$. The microphone band width is $0.3-15000 \mathrm{~Hz}$ with maximum deviation of $\pm 2.5 \%$. The pressure transducer accuracy is \pm 0.5 of full scale. Data acquisition is performed by an NI 9234 card of 4 channels, 24 bits with amplifying capability. The sampling frequency is $52.2 \mathrm{kHz}$ for each channel.

Measurements of sound are taken with $52.2 \mathrm{k}$ sample at one second. The data are analyzed by FFT. The measurements are repeated 30 times for the same run and the scheme of peak-hold is applied. Meanwhile the instantaneous volume flow rate and the downstream pressure are continuously acquired and averaged for the 30 second run. All measurements are, therefore, taken simultaneously. The raw and processed data are then stored in an Excel file sheet.

\section{Results and Discussion}

The first part in this section is attempt to use the hydraulic characteristics, and efficiency of a centrifugal pump to pre-detect the occurrence and strength of cavitation (inception, medium, or sever) at constant rotational speed 


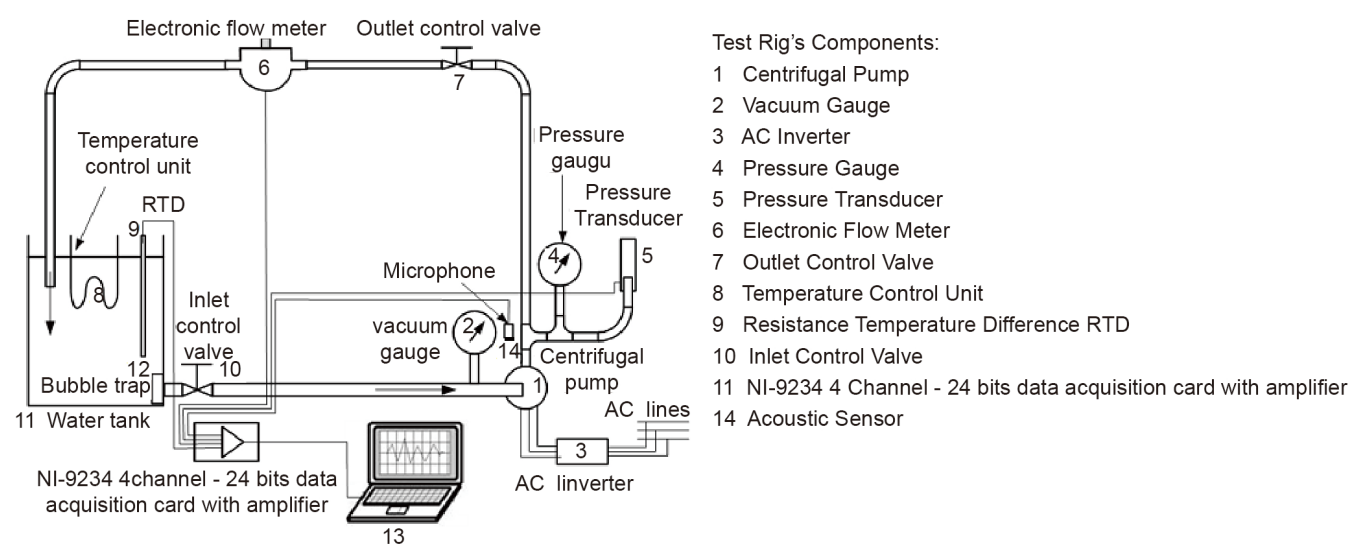

(a)

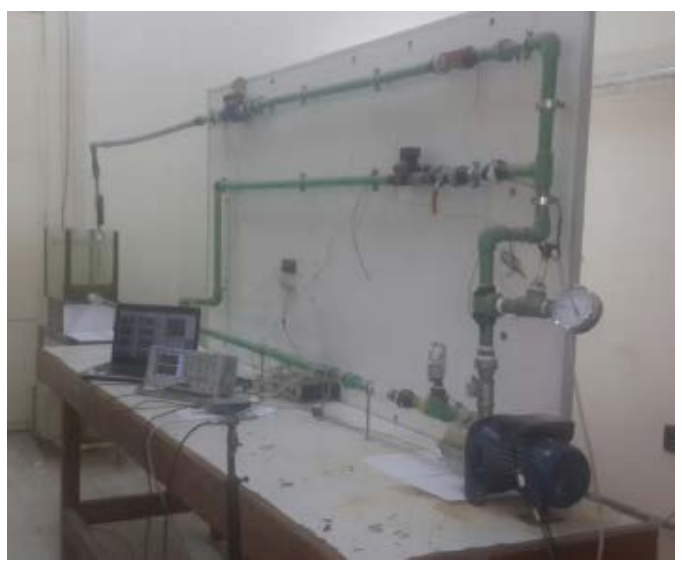

(b)

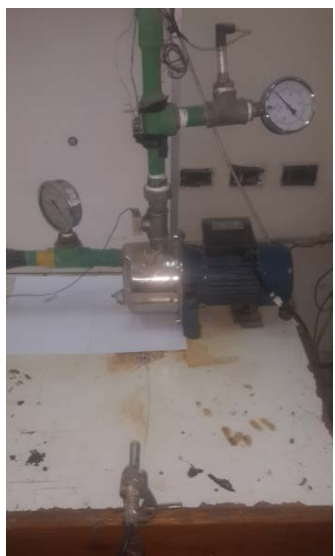

(c)

Figure 3. (a) Experimental test rig, (b) real picture of the test rig, (c) allocation of the acoustic sensor relative to the pump.

and variable suction pressures.

Figure 4 shows experimentally measured characteristic curves (discharge and total head relationship) at a rotational speed of $2850 \mathrm{rpm}(47.5 \mathrm{~Hz})$, and variable absolute values of suction pressures from $0.1 \mathrm{bar}$ to $0.78 \mathrm{bar}$. It is illustrated that, there is a definite discharge at which the performance curve separates from the conventional profile and suddenly starts to deteriorate while the flow tends to be choked (independent on head). The working point at nearly choked flow and deterioration starts is defined as the separation point, Table 1 illustrates; the discharge at separation points and the corresponding available net positive suction head for each suction pressure (according to 3\% total head drop).

The values of the available net positive suction head for the separation points listed in Table 1 are coincident with the required net positive suction head provided by the pump manufacturer as shown in Figure 4. One can deduce that; the pump starts to face cavitation from the point at which the characteristic curve separates from the conventional one and beyond. Therefore the hydraulic performance may be considered as a fist estimate for the inception of cavitation.

As an emphasis for the performance deterioration associated with the cavitation, Figure 5 shows, experimentally measured efficiency curves versus the discharge at the same previous conditions. Increasing the pump discharge increases the efficiency till its maximum value just before reaching the separation flow rate and then the efficiency decreases sharply.

The second part in this section is an attempt to detect the cavitation by measuring and analysing the sound spectrum. To limit the variation in the spectrum to the cavitation only, the mechanical and hydraulic aspects of noise have to be unified. This is achieved by applying the following constraints; constant pump rotational speed and constant pump discharge. 


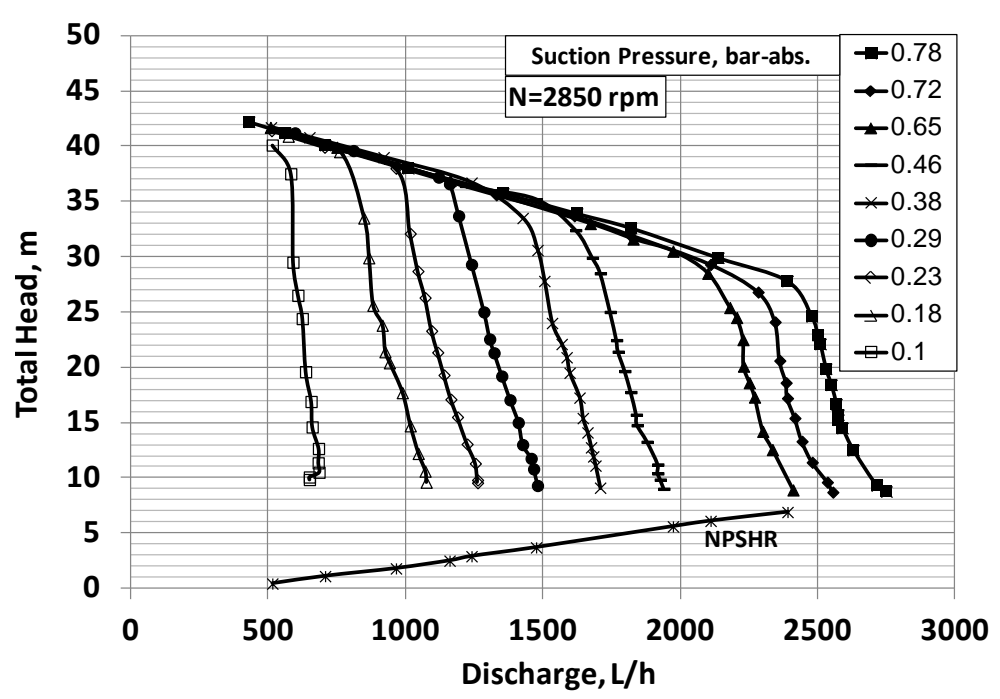

Figure 4. Pump characteristics at variable suction pressures.

Table 1. Separation point and $\mathrm{NPSH}_{\mathrm{A}}$.

\begin{tabular}{ccc}
\hline Suction Pressure bar (abs) & Discharge L/hr & NPSH $_{\mathrm{A}} \mathrm{m}$ \\
\hline 0.78 & 2390 & 6.71 \\
0.72 & 2110 & 6.25 \\
0.65 & 1970 & 5.6 \\
0.46 & 1475 & 3.87 \\
0.38 & 1240 & 3.13 \\
0.29 & 1160 & 2.23 \\
0.23 & 956 & 1.67 \\
0.18 & 707 & 1.21 \\
0.1 & 515 & 0.43 \\
\hline
\end{tabular}

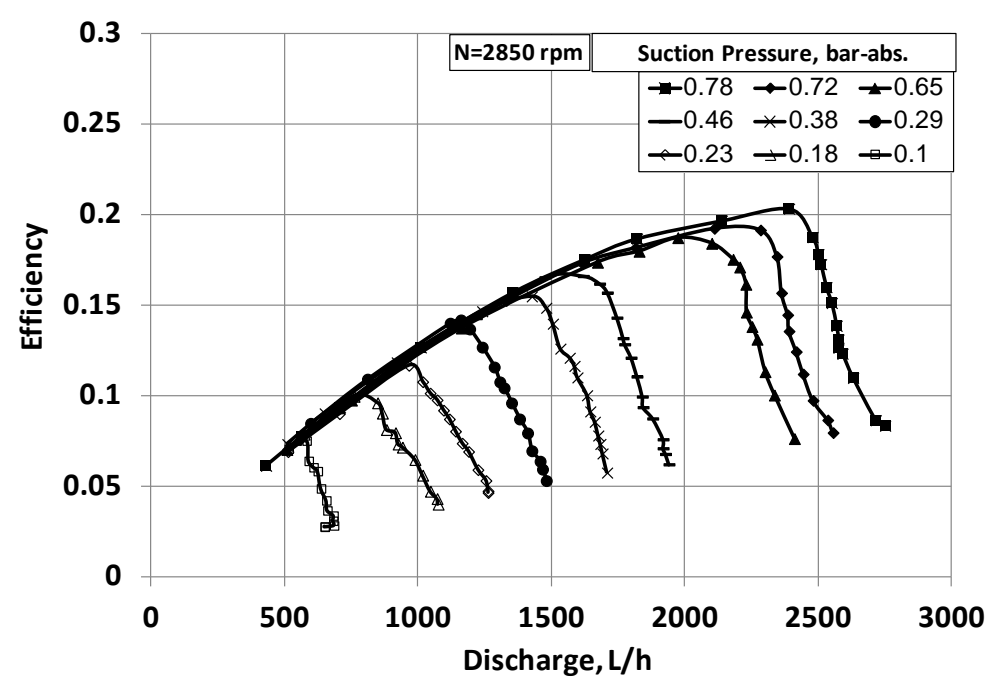

Figure 5. Pump efficiency at variable suction pressures.

In compliance with the above constraints, Figure 6 shows various sound spectrums up to $10 \mathrm{kHz}$, for five different operating cases. Each case is obtained for no-cavitation and cavitation condition at the same pump dis- 


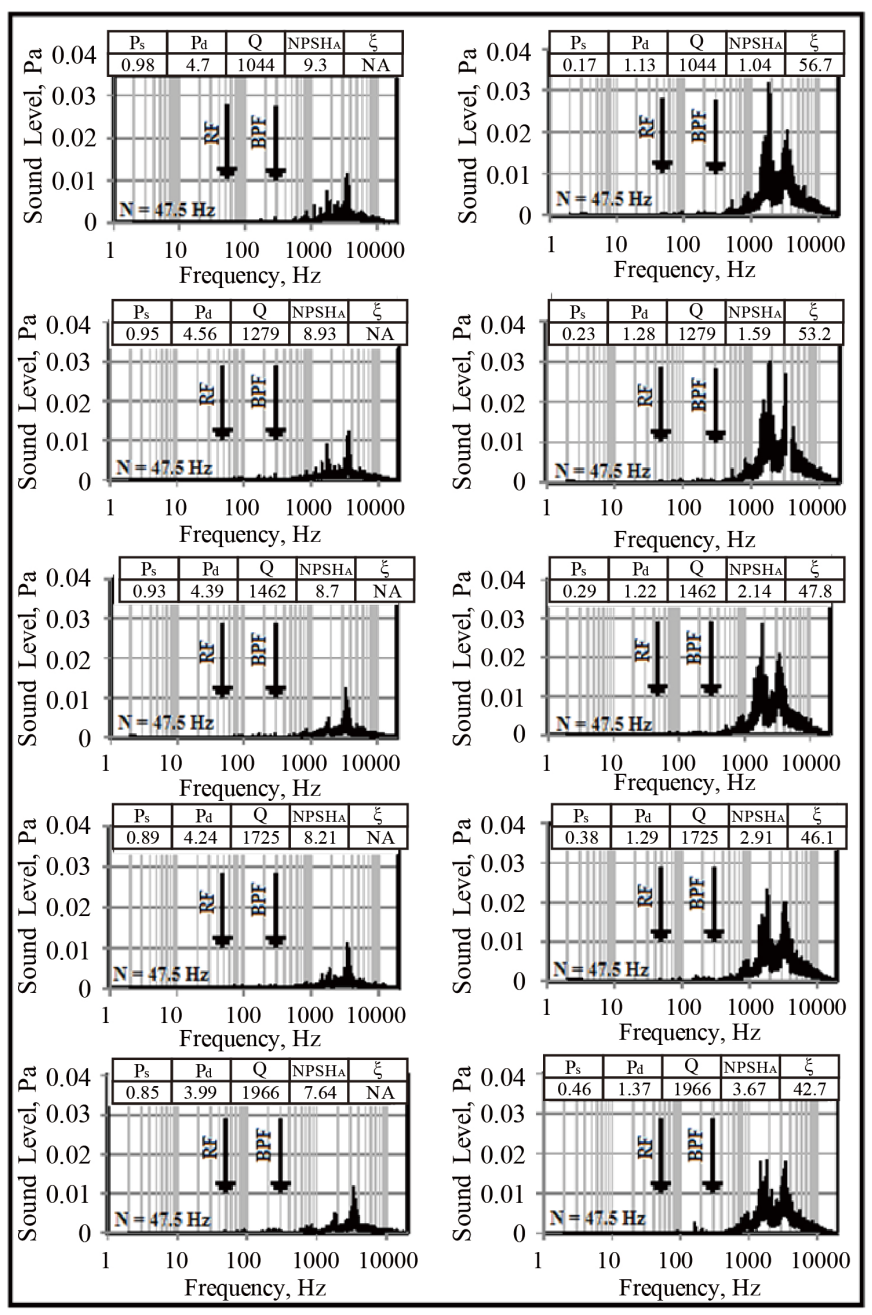

Figure 6. Sound level spectrums for non-cavitation and cavitation conditions.

charge and pump rotational speed.

Investigation the sound spectrums over the measured frequency range $(10000 \mathrm{~Hz})$, it is deduced for the discrete frequencies of RF $=47.5 \mathrm{~Hz}$, and $\mathrm{BPF}=285 \mathrm{~Hz}$ and its second, third, and fourth harmonics of $570 \mathrm{~Hz}, 855$ $\mathrm{Hz}$, and $1140 \mathrm{~Hz}$ respectively that; there are no great variations in the noise signal at these discrete frequencies for the cavitation and non-cavitation conditions.

Also, for the discrete frequency of $147 \mathrm{~Hz}$ that is mentioned in the survey as cavitation's characteristic there is no great variation in the noise signal at this frequency.

The only apparent result is that; the occurrence of cavitation results high energy signals at high frequencies from $1000 \mathrm{~Hz}$ to $10,000 \mathrm{~Hz}$.

The current question arises from this study, is the sound spectrum valid as a prediction tool for pump cavitation?

The absence of any discrete frequency to be monitored, the sound spectrum is not valid as a prediction tool for cavitation in the pumps.

\section{Conclusions}

For a centrifugal pump runs at constant speed of $2850 \mathrm{rpm}$ and variable absolute values of suction pressures from 0.1 bar to 0.78 bar, the following remarks could be concluded:

1) Sudden deterioration in the hydraulic characteristics associated with a decrease in the efficiency is a detec- 
tor for the cavitation inception.

2) Pump flow rate is almost chocked at the cavitating operating conditions.

3) At the discrete frequencies of $\mathrm{RF}=47.5$, and $\mathrm{BPF}=285 \mathrm{~Hz}$ and its second, third, and fourth harmonics of $570 \mathrm{~Hz}, 855 \mathrm{~Hz}$, and $1140 \mathrm{~Hz}$; there are no great variations in the noise signal for cavitation and non-cavitation conditions.

4) For the discrete frequency of $147 \mathrm{~Hz}$, there is no great variation in the noise signal at this frequency.

5) The occurrence of cavitation results high energy noise signals at high frequencies from $1000 \mathrm{~Hz}$ to $10,000 \mathrm{~Hz}$.

6) The absence of any discrete frequency to be monitored makes the sound spectrum not valid as a prediction tool for cavitation in the pumps.

\section{References}

[1] Tan, L., Zhu, B.S., Cao, S.L., et al. (2014) Influence of Prewhirl Regulation by Inlet Guide Vanes on Cavitation Performance of a Centrifugal Pump. Energies, 7, 1050-1065. http://dx.doi.org/10.3390/en7021050

[2] Tan, L., Zhu, B.S., Cao, S.L., et al. (2014) Numerical Simulation of Unsteady Cavitation Flow in a Centrifugal Pump at Off-Design Conditions. Proceedings of the Institution of Mechanical Engineers, Part C: Journal of Mechanical Engineering Science, 228, 1994-2006. http://dx.doi.org/10.1177/0954406213514573

[3] Kudirka, A.A. and DeCoster, M.A. (1979) Jet Pump Cavitation with Ambient and High Temperature Water. Journal of Fluids Engineering, 101, 93-99. http://dx.doi.org/10.1115/1.3448741

[4] Cudina, M. (2003) Detection of Cavitation Phenomenon in a Centrifugal Pump Using Audible Sound. Mechanical Systems and Signal Processing, 17, 1335-1347. http://dx.doi.org/10.1006/mssp.2002.1514

[5] Cudina, M. (2003) Noise as an Indicator of Cavitation in a Centrifugal Pump. Acoustical Physics, 49, 463-474. From Akusticheski Ï Zhurnal, 49, 551-564.

[6] Cudina, M. and Prezelj, J. (2009) Detection of Cavitation in Operation of Kinetic Pumps. Use of Discrete Frequency Tone in Audible Spectra. Applied Acoustics, 70, 540-546. http://dx.doi.org/10.1016/j.apacoust.2008.07.005

[7] Cudina, M. and Prezelj, J. (2009) Detection of Cavitation in Situ Operation of Kinetic Pumps: Effect of Cavitation on the Characteristic Discrete Frequency Component. Applied Acoustics Applied Acoustics, 70, 1175-1182. http://dx.doi.org/10.1016/j.apacoust.2009.04.001

[8] Cernetic, J., Prezelj, J. and Cudina, M. (2008) Use of Noise and Vibration Signal for Detection and Monitoring of Cavitation in Kinetic Pumps. The Journal of the Acoustical Society of America, 123, 3316. http://dx.doi.org/10.1121/1.2933777

[9] Cernetic, J. (2009) The Use of Noise and Vibration Signals for Detecting Cavitation in Kinetic Pumps. Proceedings of the Institution of Mechanical Engineers, Part C: Journal of Mechanical Engineering Science, 223, 1645-1655. http://dx.doi.org/10.1243/09544062JMES1404

[10] Bull, V., Civale, J., Rivens, I. and ter Haar, G.R. (2011) Cavitation Detection Using a Fibre Optic Hydrophone: A Pilot Study. AIP Conference Proceedings, 1359, 85. http://dx.doi.org/10.1063/1.3607887

[11] Baldassarre A., De Lucia, M. and Nesi, P. (1998) Real-Time Detection of Cavitation for Hydraulic Turbomachines. Real-Time Imaging, 4, 403-416.

[12] BS 5316-2:1977, ISO 3555:1977 and BS EN ISO 9906:2000. Specification for Acceptance Tests for Centrifugal, Mixed Flow and Axial Pumps. Class B Tests, MCE/6. BSI. 


\section{Nomenclature}

\begin{tabular}{|c|c|}
\hline Symbol & Definition \\
\hline BPF & Blade passing frequency, $\mathrm{Hz}$ \\
\hline$c_{1}$ & Fluid velocity at impeller inlet, m/s \\
\hline $\mathrm{C}_{1}{ }^{\prime}$ & Fluid velocity at impeller inlet corresponds to blade shift angle, m/s \\
\hline$c_{2}$ & Fluid velocity at impeller outlet, $\mathrm{m} / \mathrm{s}$ \\
\hline $\mathrm{C}_{\mathrm{u} 2}$ & Euler component of fluid velocity at impeller outlet, $\mathrm{m} / \mathrm{s}$ \\
\hline $\mathrm{C}_{\mathrm{m} 2}$ & Flow component of fluid velocity at impeller outlet, m/s \\
\hline $\mathrm{C}_{\mathrm{s}}$ & Fluid velocity at the pump suction, $\mathrm{m} / \mathrm{s}$ \\
\hline g & Gravitational acceleration, $\mathrm{m} / \mathrm{s}^{2}$ \\
\hline $\mathrm{H}$ & Pump total head, m \\
\hline Q & Pump discharge, $\mathrm{l} / \mathrm{h}$ \\
\hline $\mathrm{N}$ & Pump rotational speed, $\mathrm{Hz}$, rpm. \\
\hline $\mathrm{NPSH}_{\mathrm{A}}$ & Available net positive suction head, $\mathrm{m}$ \\
\hline $\mathrm{NPSH}_{\mathrm{R}}$ & Required net positive suction head, m \\
\hline $\mathrm{p}_{\mathrm{s}}$ & Absolute pressure in the pump suction, related to the pump centreline, $\mathrm{Pa}$ \\
\hline $\mathrm{P}_{\mathrm{s}}$ & Absolute pressure at the pump suction, bar \\
\hline$P_{d}$ & Absolute pressure at the pump discharge, bar \\
\hline $\mathrm{p}_{\mathrm{v}}$ & Absolute vapour pressure at the fluid temperature, $\mathrm{Pa}$ \\
\hline RF & Frequency corresponds to the pump rotational speed $=\mathrm{N} / 60$, rps or $\mathrm{Hz}$ \\
\hline $\mathrm{u}_{1}$ & Peripheral speed of impeller inlet, $\mathrm{m} / \mathrm{s}$ \\
\hline $\mathrm{u}_{2}$ & Peripheral speed of impeller outlet, m/s \\
\hline $\mathrm{w}_{1}$ & Fluid velocity at impeller inlet relative to the impeller peripheral speed, m/s \\
\hline $\mathrm{W}_{2}$ & Fluid velocity at impeller outlet relative to the impeller peripheral speed, $\mathrm{m} / \mathrm{s}$ \\
\hline $\mathrm{w}_{1}{ }^{\backslash}$ & $\begin{array}{l}\text { Fluid velocity at impeller inlet relative to the impeller peripheral speed corresponds to blade shift } \\
\text { angle, } \mathrm{m} / \mathrm{s}\end{array}$ \\
\hline $\mathrm{z}$ & Potential elevation, $\mathrm{m}$ \\
\hline$\beta_{1}$ & Inlet blade angle, degree \\
\hline$\beta_{1}{ }^{\prime}$ & Inlet blade angle at high flow rate, degree \\
\hline $\mathrm{B}_{2}$ & Outlet blade angle, degree \\
\hline$\Delta \beta_{1}$ & Blade shift angle at blade entrance, degree \\
\hline$\xi$ & Cavitation strength, \% \\
\hline$\rho$ & Density of fluid, $\mathrm{kg} / \mathrm{m}^{3}$ \\
\hline$\sigma$ & Thoma cavitation number \\
\hline
\end{tabular}

